\title{
On the $\mathscr{S}_{\text {semigroups of automata }}$
}

\section{Cornells Paul Vlagsma}

This thesis presents an investigation into the structure of S-semigroups of automata, a concept introduced by A.C. Fleck and S.T. Hedetniemi. As was pointed out by these authors in their original paper, this new means of associating a semigroup with an automaton gives rise to a semigroup which more faithfully carries the structure of the original automaton than does the classical semigroup.

The author of this thesis attempted to use the $S$-semigroups to determine series-parallel, and feedback decompositions of automata. This attempt was frustrated by a lack of knowledge of the structure of $S$-semigroups. Nor was it possible to determine the relation between an $S$-semigroup of an automaton and the $S$-semigroups of the automata involved in a decomposition of that automaton, for the same reason.

The usefulness of knowing the simple groups which divide a semigroup, in determining relationships between a product of semigroups and the factors of the product, and therefore the structure of a semigroup, was shown by Krohn and Rhodes, with their prime decomposition theorem for finite semigroups.

In this thesis all the simple groups which divide the S-semigroup of an automaton, $M$, are found. It is shown that these simple groups are exactly those which divide the permutation group $P_{n(M)}$, where $n(M)$ is an invariant of the automaton $M$. A brief description is given of a method of finding this invariant.

It is known that a simple group which divides a semigroup must divide a subgroup of that semigroup, and that the maximal subgroups of a semigroup

Received 13 February 1974. Thesis submitted to the Australian National University, June 1973. Degree approved, April 1974. Supervisors: Professor Hanna Neumann, Dr P.J. Cossey, Dr M.A. Ward. 
are associated with the idempotents of the semigroup. Hence, in order to find the simple groups which divide a semigroup it is only necessary to find the idempotents of the semigroup, and then the simple groups which divide the groups associated with the idempotents. This is the procedure used in this thesis.

The result of this thesis concerning the simple groups which divide the S-semigroups of automata appears to indicate that the use of these semigroups in determining the structure of automata is limited. 